\title{
Rheumatic heart disease in Indigenous children in northern Australia: differences in prevalence and the challenges of screening
}

$\mathrm{R}$ ates of acute rheumatic fever (ARF) and its sequel, rheumatic heart disease (RHD) are high in Indigenous Australians (Aboriginal Australians or Torres Strait Islander peoples). ${ }^{1,2}$ Estimates of RHD prevalence have relied on register data collected for clinical purposes or on intermittent enhanced surveillance projects, ${ }^{3}$ and have suggested that $1 \%-2 \%$ of Indigenous Australians living in northern and central Australia have RHD.

Screening for RHD provides an opportunity to accurately define the current disease burden, as well as to identify children with undiagnosed disease who may benefit from early treatment. A number of studies have shown that cardiac auscultation lacks the sensitivity and specificity required for screening for RHD and should no longer be used for this purpose. ${ }^{4-6}$ Portable echocardiography has emerged as a more valuable tool, and its usefulness was enhanced by the publication of the World Heart Federation (WHF) criteria for the echocardiographic diagnosis of RHD in 2012 (Box 1).

We recently published the results of an echocardiographic screening study of more than 5000 school-aged children, including nearly 4000 Indigenous children living in four regions of northern and central Australia. ${ }^{8}$ We used the WHF criteria to compare the echocardiographic findings of children at high and low risk of RHD (as defined by the RHD Australia guidelines ${ }^{9}$ ). We found that the overall prevalence of definite RHD in high-risk Indigenous children (8.6 per 1000) was comparable with previous register-based estimates from the Northern Territory. Definite RHD was not identified in any lowrisk non-Indigenous children.

This study is methodologically the most rigorous exploration of echocardoi: 10.5694/mja15.00139 diographic screening yet conducted,

\section{Abstract}

Objectives: To compare regional differences in the prevalence of rheumatic heart disease (RHD) detected by echocardiographic screening in high-risk Indigenous Australian children, and to describe the logistical and other practical challenges of RHD screening.

Design: Cross-sectional screening survey performed between September 2008 and November 2010.

Setting: Thirty-two remote communities in four regions of northern and central Australia.

Participants: 3946 Aboriginal or Torres Strait Islander children aged $5-15$ years.

Intervention: Portable echocardiography was performed by cardiac sonographers. Echocardiograms were recorded and reported offsite by a pool of cardiologists.

Main outcome measures: RHD was diagnosed according to 2012 World Heart Federation criteria.

Results: The prevalence of definite RHD differed between regions, from 4.7/1000 in Far North Queensland to 15.0/1000 in the Top End of the Northern Territory. The prevalence of definite RHD was greater in the Top End than in other regions (odds ratio, 2.3; 95\% Cl, 1.2-4.6, $P=0.01$ ). Fifty-three per cent of detected cases of definite RHD were new cases; the prevalence of new cases of definite RHD was 4.6/1000 for the entire sample and 7.0/1000 in the Top End. Evaluation of socioeconomic data suggests that the Top End group was the most disadvantaged in our study population.

Conclusions: The prevalence of definite RHD in remote Indigenous Australian children is significant, with a substantial level of undetected disease. Important differences were noted between regions, with the Top End having the highest prevalence of definite RHD, perhaps explained by socioeconomic factors. Regional differences must be considered when evaluating the potential benefit of widespread echocardiographic screening in Australia.

and the first cross-sectional survey of the prevalence of RHD in Australia. However, we did not report the data in sufficient detail to maximise its relevance for local RHD control in Australia. In this article, we describe the prevalence of definite and borderline RHD in Indigenous children from the Top End of the NT, Central Australia, Far North Queensland (FNQ), including the Torres Strait, and the Kimberley region of Western Australia. By comparing the findings in different regions and describing some of the challenges of the screening process, we aim to inform decision making about the potential impact and usefulness of echocardiographic screening for RHD in different Australian regions.

\section{Methods}

\section{Design, setting and participants}

The study design and population and the sample size calculation have been described previously. ${ }^{8}$ Briefly, we performed screening echocardiograms on 3946 Indigenous children aged 5-15 years living in remote communities in northern Australia. Thirty-two communities were selected from four geographical regions (Box 2). Children were identified by the enrolment records of 
participating schools and were recruited at school or by approaching their families. Written informed consent was obtained from parents or guardians, and written consent was also obtained from children who were at least 13 years old.

The study was conducted from September 2008 to November 2010. Ethics approval was obtained from the Human Research Ethics Committee of the Northern Territory Department of Health and Community Services, the Central Australian Human Research Ethics Committee, the Cairns and Hinterland Health Service District Human Research Ethics Committee, the James Cook University Human Ethics Committee, the University of Western Australia Human Research Ethics Committee, and the Western Australian Aboriginal Health Information and Ethics Committee.

\section{Echocardiography protocol, reporting and definitions}

Screening echocardiograms were performed by cardiac sonographers according to an abbreviated protocol that focused on the mitral and aortic valves. Sonographers were provided with a list of features that prompted a more detailed, comprehensive echocardiogram, also performed at the time of screening, if required. Screening echocardiograms were recorded to DVD and reported offsite by a pool of 14 cardiologists according to our standardised electronic protocol. These data were used post hoc to determine whether children met the WHF definitions of definite or borderline RHD.

\section{Clinical follow-up}

Separate to reporting for research purposes, all comprehensive echocardiograms were sent to a local cardiologist to guide clinical management of the participant. The cardiologist provided a written report that included the echocardiographic findings and recommendations for follow-up, including secondary prophylaxis. Reports were sent to the primary health care team, who used existing clinical services to coordinate the necessary referrals.

1 Echocardiographic criteria for rheumatic heart disease (RHD) in individuals aged $\leq 20$ years

Definite RHD (one of the following features):

- Pathological mitral regurgitation and at least two morphological features of $\mathrm{RHD}$ of the mitral valve;

- Mitral stenosis mean gradient $\geq 4 \mathrm{~mm} \mathrm{Hg}$;*

- Pathological aortic regurgitation and at least two morphological features of RHD of the aortic valve; ${ }^{\dagger}$

- Borderline disease of both the aortic valve and mitral valve. ${ }^{\ddagger}$

Borderline RHD (one of the following features):

- At least two morphological features of RHD of the mitral valve without pathological mitral regurgitation or mitral stenosis;

- Pathological mitral regurgitation;

- Pathological aortic regurgitation.

* Congenital mitral valve anomalies must be excluded. † Bicuspid aortic valve, dilated aortic root and hypertension must be excluded. $\ddagger$ Combined aortic and mitral regurgitation in high prevalence regions and in the absence of congenital heart disease is regarded as rheumatic. The four Doppler echocardiographic criteria for pathological mitral regurgitation are that it be seen in two views; in at least one view, jet length $\geq 2 \mathrm{~cm}$; velocity $\geq 3 \mathrm{~m} / \mathrm{s}$ for one complete envelope; and pan-systolic jet in at least one envelope. The criteria for pathological aortic regurgitation are that it be seen in two views; in at least one view, jet length $\geq 1 \mathrm{~cm}$; velocity $\geq 3 \mathrm{~m} / \mathrm{s}$ in early diastole; and pan-diastolic jet in at least one envelope). Adapted from Reményi et al. ${ }^{7}$

2 Northern Australian sites where echocardiographic screening for rheumatic heart disease was undertaken for this study
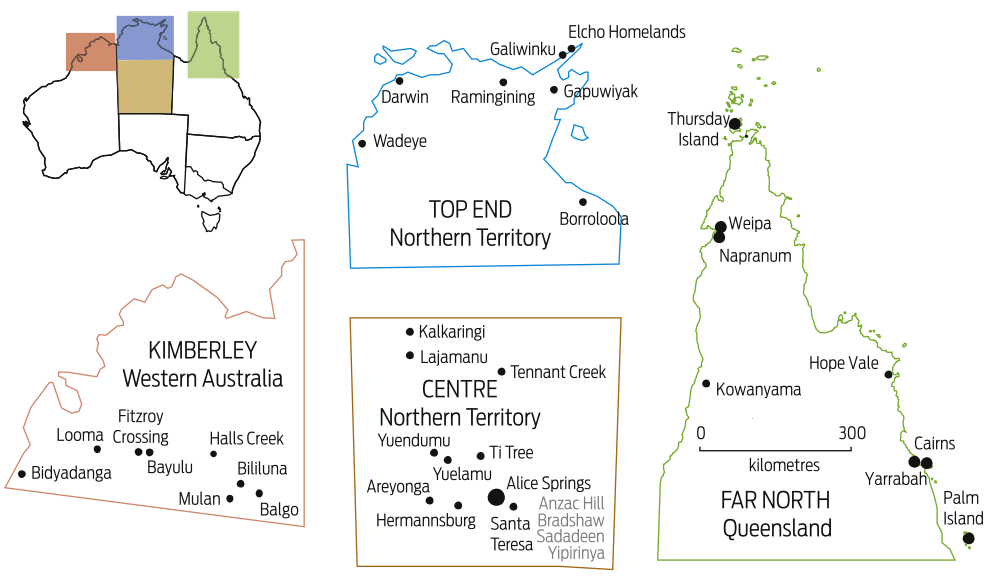

\section{Socioeconomic comparisons}

We explored whether differences in RHD prevalence between regions could be attributed to socioeconomic or demographic factors. No information about socioeconomic factors was collected from individual participants. Instead, we used publicly available statistics to compare the socioeconomic characteristics of the participating schools and communities. Information about school attendance and the Indigenous status of enrolled students, as well as Index of Community Socio-Educational
Advantage $\left(\mathrm{ICSEA}^{10}\right)$ scores were obtained for each participating school from the Australian Government's MySchool website. ${ }^{11}$ The ICSEA is a measure of the educational advantage of the students enrolled at a particular school, based on information about each student's family background (including parental occupation and level of education). The median value of the scale is 1000 with an SD of 100 .

Information about household crowding and Socio-Economic Indexes 
for Areas $\left(\mathrm{SEIFA}^{12}\right)$ scores were obtained for each participating community from the Australian Bureau of Statistics 2011 census data. ${ }^{13}$ Two SEIFA scores were analysed: the Index of Relative Social Disadvantage (IRSD) and the Index of Relative Social Advantage and Disadvantage (IRSAD). These indices summarise socioeconomic information about the people and households in a geographical area, and scales are standardised with a mean value of 1000 and an SD of 100.

ICSEA, IRSD and IRSAD scores were assigned to individuals according to their school or community and to calculate aggregate scores for each of the four study regions.

\section{Statistical analysis}

Statistical analysis was performed with the Stata statistical package (version 12.1; StataCorp). Descriptive data are presented as medians and interquartile range (IQR) for nonnormally distributed variables. Medians were compared with the Mann-Whitney $U$ test (for two groups) or the Kruskal-Wallis test (for more than two groups). Categorical variables were compared with the $\chi^{2}$ test. RHD prevalence (with 95\% CIs) was calculated for the entire study sample and for each of the four regions. Multivariate logistic regression was used to compare the proportion of children with RHD in each region. Socioeconomic variables were compared by means of ANOVA (IRSAD, IRSD and ICSEA) or Kruskal-Wallis and Mann-Whitney $U$ tests (household crowding).

\section{Results}

The demographic characteristics of the 3946 remote Indigenous children who had a screening echocardiogram are presented in Box 3. Forty-one per cent of the FNQ participants were identified as Torres Strait Islanders or Aboriginal and Torres Strait Islanders, whereas more than $99 \%$ of the other groups were identified as Aboriginal only.

Despite the similar age and sex distribution of all four groups, children from the Top End of the NT had a significantly lower median body weight and body mass index than children from the other three regions (compared with Central Australia and FNQ, $P<0.001$; with the Kimberley, $P=0.004$; Box 3).

Of the 569 comprehensive echocardiograms performed $(13.3 \%$ of children screened), significantly more were undertaken in FNQ $(17.2 \%)$ than in other jurisdictions
$(P<0.001$ compared with the Kimberley, $P<0.001$; with Central Australia, $P=0.002$; with the Top End, $P=0.26$; Box 3 ). In the FNQ group, more Torres Strait Islander children $(20.4 \%)$ required a comprehensive echocardiogram than did non-Torres Strait Islander children $(14.9 \%, P<0.001)$.

\section{Prevalence of RHD based on the WHF criteria}

The prevalence of definite and borderline RHD in each region is presented in Box 4 . The prevalence of definite RHD was higher in Top End children than in children from the three other jurisdictions combined (odds ratio [OR], 2.3; 95\% CI, 1.2-4.6, $P=0.01)$. This difference was not observed in the borderline RHD category.

We have previously reported that 18 of the 34 children $(52.9 \%)$ who met the criteria for definite RHD were new cases (no previous history of ARF or RHD); ${ }^{8}$ the majority $(93.9 \%)$ of children meeting the criteria for borderline RHD were also new cases. The prevalence of previously undiagnosed definite RHD detected in the entire study sample by screening was 4.6 per 1000 (95\% CI, 2.7-7.2); for the Top End, the prevalence of new cases of definite RHD was 7.0 per 1000 (95\% CI, 2.8-14.4).

3 Demographic characteristics of Indigenous children screened for rheumatic heart disease

\begin{tabular}{|c|c|c|c|c|c|}
\hline Characteristic & $\begin{array}{l}\text { Top End } \\
(n=1000)\end{array}$ & $\begin{array}{l}\text { Central Australia } \\
\quad(n=895)\end{array}$ & $\begin{array}{l}\text { Far North Queensland } \\
\qquad(n=1265)\end{array}$ & $\begin{array}{l}\text { Kimberley } \\
(n=786)\end{array}$ & $P$ \\
\hline \multicolumn{6}{|l|}{ Sex, $n(\%)$} \\
\hline Male & $497(49.7 \%)$ & 479 (53.5\%) & $641(50.7 \%)$ & 389 (49.5\%) & $0.30 *$ \\
\hline Female & $503(50.3 \%)$ & $416(46.5 \%)$ & $624(49.3 \%)$ & 397 (50.5\%) & \\
\hline \multicolumn{6}{|l|}{ Ethnicity, $n(\%)$} \\
\hline Aboriginal & 998 (99.8\%) & $892(99.7 \%)$ & $746(59.0 \%)$ & $786(100.0 \%)$ & \\
\hline Torres Strait Islander & $2(0.2 \%)$ & $2(0.2 \%)$ & $303(24.0 \%)$ & 0 & $<0.001 *$ \\
\hline $\begin{array}{l}\text { Aboriginal and Torres } \\
\text { Strait Islander }\end{array}$ & 0 & $1(0.1 \%)$ & $216(17.1 \%)$ & 0 & \\
\hline $\begin{array}{l}\text { Comprehensive } \\
\text { echocardiogram } \\
\text { performed, } n(\%)\end{array}$ & $153(15.3 \%)$ & 111 (12.4\%) & $217(17.2 \%)$ & $88(11.2 \%)$ & $<0.001^{*}$ \\
\hline Age (years), median (IQR) & $9.4(7.4-11.6)$ & $9.3(7.3-11.3)$ & $9.2(7.2-11.2)$ & $9.3(7.3-11.5)$ & $0.15^{\dagger}$ \\
\hline Weight (kg), median (IQR) & $26.5(21.1-35.5)$ & $29.8(22.9-40.8)$ & $28.5(21.8-39.5)$ & $27.4(21.7-39.0)$ & $<0.001^{\dagger}$ \\
\hline Height (cm), median (IQR) & 133.0 (121.9-147.0) & $135.0(123.0-149.0)$ & $133.4(120.1-145.8)$ & $133.0(121.4-148.2)$ & $0.01^{\dagger}$ \\
\hline BMI $\left(\mathrm{kg} / \mathrm{m}^{2}\right)$, median (IQR) & $15.1(14.0-16.6)$ & $16.2(14.9-18.9)$ & $16.2(14.7-19.0)$ & $15.7(14.4-17.9)$ & $<0.001^{\dagger}$ \\
\hline
\end{tabular}

$\mathrm{BMI}=$ body mass index; IQR $=$ interquartile range. $* \chi^{2}$ test; $\uparrow$ Kruskal-Wallis test. $\bullet$ 


\begin{tabular}{|c|c|c|c|c|c|c|}
\hline & Top End & $\begin{array}{c}\text { Central } \\
\text { Australia }\end{array}$ & $\begin{array}{l}\text { Far North } \\
\text { Queensland }\end{array}$ & Kimberley & Total & $P\left(\chi^{2}\right)$ \\
\hline \multicolumn{7}{|l|}{ Definite RHD } \\
\hline New cases & 7 & 4 & 5 & 2 & 18 & \multirow{4}{*}{0.06} \\
\hline Known cases & 8 & 2 & 1 & 5 & 16 & \\
\hline Prevalence & 15.0/1000 & $6.7 / 1000$ & $4.7 / 1000$ & $8.9 / 1000$ & 8.6/1000 & \\
\hline $95 \% \mathrm{Cl}$ & $8.4-24.6$ & $2.5-14.5$ & $1.7-10.2$ & $3.6-18.2$ & $6.0-12.0$ & \\
\hline \multicolumn{7}{|l|}{ Borderline RHD } \\
\hline New cases & 17 & 14 & 23 & 8 & 62 & \multirow{4}{*}{0.41} \\
\hline Known cases & 1 & 1 & 2 & 0 & 4 & \\
\hline Prevalence & 18.0/1000 & $16.8 / 1000$ & 19.8/1000 & $10.2 / 1000$ & 16.7/1000 & \\
\hline $95 \% \mathrm{Cl}$ & 10.7-28.3 & $9.4-27.5$ & $12.8-29.0$ & $4.4-20.0$ & $13.0-21.2$ & \\
\hline Total screened & 1000 & 895 & 1265 & 786 & 3946 & \\
\hline
\end{tabular}

\section{Comparison of the socioeconomic profiles of the four regions}

Thirty-eight schools from 32 communities participated in the screening study. Mean and median ICSEA, IRSD and IRSAD scores for each region are presented in Box 5 . The Top End communities had significantly lower mean ICSEA, IRSD and IRSAD scores (ANOVA), and significantly higher levels of household crowding (KruskalWallis, Mann-Whitney $U$ tests) than the other regions $(P<0.05$ for all comparisons; Top End versus other regions combined or individually). Top End schools also had significantly lower median ICSEA scores than the other regions combined and each of Central Australia and FNQ (for each comparison, $P<0.001$ ), but not when compared with Kimberley schools $(P=0.43)$.

\section{Discussion}

This is the first prospective screening survey for RHD in Indigenous Australian children, and the first study to provide reliable information about the epidemiology of RHD in children from FNQ and the Kimberley region of Western Australia. Our previous report confirmed that the prevalence of RHD is high in Indigenous children, and that the overall prevalence of definite RHD in school-aged children (8.6 per 1000) is comparable with figures from developing countries. ${ }^{14-18}$ Although this figure is similar to previous estimates of the prevalence of RHD in the NT, ${ }^{1,2,19}$ there are important differences between the four regions when examined individually.

The most striking difference is the higher prevalence of definite RHD in children from the Top End of the NT. The prevalence of 15.0 per 1000 is two to three times higher than in other regions, and nearly triple the previously published estimates of RHD prevalence in Top End children (5.8 per $1000^{20}$ ). Two more recent audits of the NT register have been undertaken, but only the combined data from the Top End and Central Australia have been published, ${ }^{1,19}$ reporting an RHD prevalence of 8.5 per 1000 in Indigenous children aged 5-14 years in the NT. Our study suggests that this significantly underestimates the burden of disease in the Top End, and that disease epidemiology may be different in the Top End and Central Australia.

This difference has not previously been reported, and reasons for a higher disease burden in the Top End are not clear. However, some features of our study sample may be relevant. We noted that the growth parameters of Top End children were significantly lower than those of children in the other regions, and that the participating Top End communities had the highest number of people per household, a mean of 6.3 persons, compared with the Australian average of 2.6 persons per household. ${ }^{12}$ In addition, the ICSEA, ISRD and IRSAD scores were also lowest in our Top End sample, between three and five SDs below the Australian average. It was striking how far below the Australian mean these scores were in all regions, highlighting the extreme disadvantage experienced in remote Aboriginal communities. We attempted to quantify the relationship between definite RHD and the four socioeconomic measures by logistic regression, but the small number of cases of definite RHD prevented this.

These observations suggest that the participating communities from the Top End were the most disadvantaged of the remote Indigenous communities we surveyed. Given that poverty-related factors, such as overcrowded housing, are known to be significant risk factors for ARF and RHD, ${ }^{21-23}$ extreme disadvantage would provide a plausible explanation for the higher prevalence of RHD in the Top End. Other possibilities include inherent differences in host susceptibility or in circulating strains of group A Streptococcus (GAS), but data are not available for the four sampled regions to explore these hypotheses. One NT study that investigated the diversity of GAS strains in the NT did not find 
5 Comparison of the socioeconomic characteristics of the four screening regions

\begin{tabular}{|c|c|c|c|c|}
\hline & Top End & Central Australia & $\begin{array}{l}\text { Far North } \\
\text { Queensland }\end{array}$ & Kimberley \\
\hline $\begin{array}{l}\text { Number of Indigenous children aged 5-14 years } \\
\text { who were screened }\end{array}$ & 1000 & 895 & 1265 & 786 \\
\hline Number of participating communities & 7 & 10 & 7 & 8 \\
\hline Number of participating schools & 7 & 14 & 8 & 9 \\
\hline $\begin{array}{l}\text { Estimated number of Indigenous students enrolled } \\
\text { in participating schools (all ages)* }\end{array}$ & 1765 & 1744 & 2635 & 1250 \\
\hline $\begin{array}{l}\text { Estimated percentage of enrolled Indigenous } \\
\text { students who were screened }\end{array}$ & $56.7 \%$ & $51.3 \%$ & $48.0 \%$ & $62.9 \%$ \\
\hline $\begin{array}{l}\text { Average school attendance in participating } \\
\text { schools }^{11}\end{array}$ & $65.0 \%$ & $68.0 \%$ & $79.0 \%$ & $67.0 \%$ \\
\hline \multicolumn{5}{|l|}{ ICSEA score of participating schools ${ }^{17}$} \\
\hline Mean (SD) & $576(38)$ & $643(79)$ & $622(80)$ & $583(48)$ \\
\hline Median (IQR) & $569(556-590)$ & $631(566-712)$ & $587(581-592)$ & $567(557-612)$ \\
\hline \multicolumn{5}{|l|}{ IRSAD score of participating communities ${ }^{12}$} \\
\hline Mean (SD) & $631(67)$ & $734(96)^{\dagger}$ & $759(180)$ & $711(28)$ \\
\hline Median (IQR) & $688(580-690)$ & $695(655-831)$ & $678(644-913)$ & $694(694-758)$ \\
\hline \multicolumn{5}{|l|}{ IRSD score of participating communities ${ }^{12}$} \\
\hline Mean (SD) & $533(104)$ & $676(122)^{\dagger}$ & $712(224)$ & $650(33)$ \\
\hline Median (IQR) & $606(443-641)$ & $618(570-795)$ & $621(585-903)$ & $628(628-704)$ \\
\hline \multicolumn{5}{|l|}{$\begin{array}{l}\text { Number of people per household in participating } \\
\text { communities }^{12}\end{array}$} \\
\hline Mean (SD) & $6.3(0.9)$ & $4.8(1.1)^{\dagger}$ & $4.5(0.5)$ & $4.8(0.7)$ \\
\hline Median (IQR) & $6.8(5.1-7.0)$ & $5.0(4.1-5.9)$ & $4.2(4.0-5.0)$ & $4.6(4.3-5.6)$ \\
\hline
\end{tabular}

"NT-endemic" strains, and the authors concluded that the high burden of GAS disease was more probably related to poor living conditions than to bacterial factors. ${ }^{24}$

Selection bias may also contribute to the observed differences in RHD prevalence. Given the logistical challenges of surveying a large number of Indigenous children in remote areas, we were unable to select communities at random. We instead carefully selected communities of different sizes and from different areas in the same geographic region to provide as broad a sample as possible (Box 2).

Only about $50 \%$ of school-enrolled children were screened in our study (although the percentages in Box 5 are slight underestimates, because the school enrolment record includes children of all ages, some of whom were not eligible for our study).
Given that the average daily attendance in participating schools was $69 \%$, this result is understandable, and indicates our efforts to maximise recruitment.

Whether the children we screened were representative of all children in the participating communities is an important question. We were unable to collect information about children who had not consented to the study, but Box 3 shows that there were no differences in the sex or age distributions of the samples from each region. It is probable that these figures (equal sex and normal age distributions) are representative of the communities as a whole, and that selection bias is unlikely to explain the observed differences in RHD prevalence.

However, selection bias may have resulted in an overall underestimation of RHD prevalence. A school- based approach to screening is practical, but potentially excludes those most at risk of disease, such as children who are too sick to attend school, or who live in the most marginalised families. This may have resulted in underestimation of the full burden of RHD in remote Indigenous communities.

The number of new cases detected is a crucial element in evaluating the usefulness of any screening program. More than half of the children meeting the criteria for definite RHD were new cases (Box 4), with an overall prevalence of 4.6 new cases per 1000 children screened. This figure was substantially higher in the Top End cohort, and our results suggest that for every 1000 Top End children screened, 7 new cases of definite RHD would be detected, equivalent to about 50 new cases in this population. This information is 
critical for evaluating the costeffectiveness of screening, and we are currently analysing the data.

We encountered a number of practical difficulties that have implications for future echocardiographic screening in remote Australia. The logistical challenges of travel to remote communities are clear; travel by road is slow and sometimes impossible, and travel by plane is expensive, requiring chartered flights to isolated areas not served by commercial flights. After staff had arrived in the communities, the biggest challenge was finding and obtaining consent from the children to be screened, as school attendance was poor. We tried to include absentees by extending our screening activities beyond the school grounds, which was time-consuming and inefficient.

The most significant challenges faced by this study related to clinical follow-up and communication with families and health care providers. A total of 569 children $(14.4 \%$, Box 3) had comprehensive echocardiograms that required timely review by an offsite cardiologist to guide clinical management. This considerably increased the workload of local cardiologists, and it frequently took weeks to months for reports to be completed. Once available, the reports themselves often generated confusion and frustration for health care providers, as illustrated by a qualitative survey of health care providers in three participating screening sites. ${ }^{25}$ The WHF diagnostic criteria had not yet been published when our study commenced, so there was uncertainty about the significance of minor echocardiographic changes in an otherwise healthy child. This resulted in many paediatric cardiology referrals, which often challenged the capacity of local services. ${ }^{25}$ If echocardiographic screening is to become feasible as a routine approach, a technical aspect that must be refined is thus to reduce the number of comprehensive echocardiograms that require review by a cardiologist. Ensuring that health systems are equipped to deal with the additional increase in case numbers is vital before initiating routine screening activity. $^{26}$

The impact of screening on the families of 68 children in our study was explored by Wark and colleagues with a Quality of Life (QOL) questionnaire. ${ }^{25}$ Although there was no difference in the overall QOL summary scores, carers of children with possibly abnormal echocardiograms had poorer QOL scores in subscales pertaining to general health perception and parental emotional impact. In contrast, a study by a New Zealand group $^{27}$ surveyed 114 families who had participated in a more recent school-based echocardiographic screening program, and found unanimous support for the program. The authors concluded that the screening process had no negative effects, nor were there short-term adverse effects in the families of children with abnormal results, in terms of either health perception or of parental anxiety.

The timing of the two studies and methodological differences may explain these contrasting findings. In the New Zealand study, screening and reporting occurred within a much shorter time period, and clinical follow-up was performed by clinicians who were directly involved in the research process. In addition, the
WHF criteria had been published before the study commenced, reducing diagnostic uncertainty regarding the significance of minor echocardiographic abnormalities and facilitating appropriate clinical follow-up.

In summary, our study identified a previously unrecognised difference in the prevalence of RHD in four remote regions of northern Australia. The prevalence of definite RHD in Top End children was nearly twice as high as that in the other three regions, and this may be related to socioeconomic factors. We estimate that $4-8$ per 1000 Indigenous children in remote communities have undetected RHD that could be identified by echocardiographic screening. Whether such screening should be recommended will require further and careful consideration of its costeffectiveness, feasibility, sustainability and impact on primary and specialist health care services. We are currently preparing a costeffectiveness analysis that will allow us to make informed recommendations regarding RHD screening to national policymakers.

Acknowledgements: We gratefully acknowledge the work of study staff Loraine Kelpie, Vijaya Joshi, Colette Davis, Rhona Dawson and Yvonne Hodder. We also thank Josh Sher and the other echocardiographers, Cabrini Health, and cardiologist Krishna Kumar; this work would not have been possible without their generous support. We also thank the staff and students at the participating schools and health clinics. This study was supported by the Office of Aboriginal and Torres Strait Islander Health of the Australian Government. Additional funding was received from the Children First Foundation, Kiwanis International, and Cabrini Health. Kathryn Roberts received an Australian Postgraduate Award Scholarship from the Charles Darwin University. Graeme Maguire is supported by an NHMRC Practitioner Fellowship and the Margaret Ross Chair in Indigenous Health.

\section{Competing interests: No relevant disclosures.}

(c) 2015 AMPCo Pty Ltd. Produced with Elsevier B.V. All rights reserved. 
1 Lawrence JG, Carapetis JR, Griffiths K, et al. Acute rheumatic fever and rheumatic heart disease: incidence and progression in the Northern Territory of Australia, 1997 to 2010. Circulation 2013; 128: 492-501.

2 Australian Institute of Health and Welfare. Rheumatic heart disease and acute rheumatic fever in Australia: 1996-2012. Canberra: AlHW, 2013. (Cat. No. CVD 60.) http://www.aihw.gov.au/ publication-detail/?id=60129542750 (accessed Jun 2015).

3 Rémond MG, Severin KL, Hodder Y, et al. Variability in disease burden and management of rheumatic fever and rheumatic heart disease in two regions of tropical Australia. Intern Med J 2013; 43: 386-393.

4 Roberts KV, Brown ADH, Maguire GP, et al. Utility of auscultatory screening for detecting rheumatic heart disease in high-risk children in Australia's Northern Territory. Med J Aust 2013; 199: 196-199. https://www.mja.com.au/ journal/2013/199/3/utilityauscultatory-screening-detectingrheumatic-heart-disease-high-risk

5 Marijon E, Tafflet M, Jouven X. Time to use ultrasound and not stethoscopes for rheumatic heart disease screening. Nat Clin Pract Cardiovasc Med 2008; 5: El-E3.

6 Carapetis JR, Hardy M,

Fakakovikaetau T, et al. Evaluation of a screening protocol using auscultation and portable echocardiography to detect asymptomatic rheumatic heart disease in Tongan schoolchildren. Nat Clin Pract Cardiovasc Med 2008; 5 : 411-417.

7 Reményi B, Wilson N, Steer A, et al. World Heart Federation criteria for echocardiographic diagnosis of rheumatic heart disease - an evidence-based guideline. Nat Rev Cardiol 2012; 9: 297-309.

8 Roberts K, Maguire G, Brown A, et al. Echocardiographic screening for rheumatic heart disease in high and low risk Australian children. Circulation 2014; 129: 1953-1961.

9 RHD Australia (ARF/RHD writing group), National Heart Foundation of Australia, Cardiac Society of Australia and New Zealand. The Australian guideline for prevention, diagnosis and management of acute rheumatic fever and rheumatic heart disease (2nd edition). 2012. http://www. rhdaustralia.org.au/sites/default/files/ guideline_0.pdf (accessed Jun 2015).

10 Australian Curriculum and Assessment Reporting Authority. MySchool. Guide to understanding ICSEA. Sydney: ACARA, 2012. http://www.saasso.asn. au/wp-content/uploads/2012/08/ Guide_to_understanding_ICSEA.pdf (accessed Dec 2014).

11 Australian Curriculum and Assessment Reporting Authority. MySchool [website]. http://www.myschool.edu. au/ (accessed Dec 2014).

12 Australian Bureau of Statistics. Census of Population and Housing: SocioEconomic Indexes for Areas (SEIFA), 2011. Canberra: ABS, 2013. (ABS Cat. No. 2033.0.55.001.) http://www.abs. gov.au/ausstats/abs@.nsf/Lookup/2 033.0.55.001main+features 42011 (accessed Dec 2014).

13 Australian Bureau of Statistics. Census [2011]. Data \& analysis. http://www.abs. gov.au/websitedbs/censushome.nsf/ home/data?opendocument\&navpos $=2$ 00. (accessed Dec 2014).

14 Baroux N, Rouchon B, Huon B, et al. High prevalence of rheumatic heart disease in schoolchildren detected by echocardiography screening in New Caledonia. J Paediatr Child Health 2013; 49: 109-114.

15 Beaton A, Okello E, Lwabi P, et al. Echocardiography screening for rheumatic heart disease in Ugandan schoolchildren. Circulation 2012; 125: 3127-3132.

16 Mirabel M, Celermajer DS, Ferreira B, et al. Screening for rheumatic heart disease: evaluation of a simplified echocardiography-based approach. Eur Heart J Cardiovasc Imaging 2012; 13: 1024-1029.

17 Paar JA, Berrios NM, Rose JD, et al. Prevalence of rheumatic heart disease in children and young adults in Nicaragua. Am J Cardiol 2010; 105: 1809-1814.

18 Colquhoun SM, Kado JH, Remenyi B, et al. Echocardiographic screening in a resource poor setting: borderline rheumatic heart disease could be a normal variant. Int J Cardiol 2014; 173: 284-289.

19 Parnaby MG, Carapetis JR. Rheumatic fever in Indigenous Australian children. J Paediatr Child Health 2010; 46: 527-533.
20 Australian Institute of Health and Welfare: Field B. Rheumatic heart disease: all but forgotten in Australia except among Aboriginal and Torres Strait Islander peoples. Canberra: AlHW, 2004. (AlHW Cat. No. AUS 48.) http://www.aihw.gov.au/publicationdetail/?id=6442467621 (accessed Jun 2015).

21 Jaine R, Baker M, Venugopal K. Acute rheumatic fever associated with household crowding in a developed country. Pediatr Infect Dis J 2011; 30: 315-319.

22 Steer AC, Carapetis JR, Nolan TM, Shann F. Systematic review of rheumatic heart disease prevalence in children in developing countries: the role of environmental factors. J Paediatr Child Health 2002; 38: 229-234.

23 Brown A, McDonald Ml, Calma T. Rheumatic fever and social justice. Med J Aust 2007; 186: 557-558. https:// www.mja.com.au/search/site/ Rheumatic\%20fever\%20and\% 20social\%20justice

24 Towers RJ, Carapetis JR, Currie BJ, et al. Extensive diversity of Streptococcus pyogenes in a remote human population reflects global-scale transmission rather than localised diversification. PLOS One 2013; 8: e73851.

25 Wark EK, Hodder YC, Woods CE, Maguire GP. Patient and health-care impact of a pilot rheumatic heart disease screening program. J Paediatr Child Health 2013; 49: 297-302.

26 Roberts K, Colquhoun S, Steer A, et al. Screening for rheumatic heart disease: current approaches and controversies. Nat Rev Cardiol 2012; 10: 49-58.

27 Perelini F, Blair N, Wilson N, et al. Family acceptability of school-based echocardiographic screening for rheumatic heart disease in a high-risk population in New Zealand. $J$ Paediatr Child Health 2015; 51: 682-688. 